\title{
SYNTHESIS AND CHARACTERIZATION OF AMINATED COPOLYMERS OF POLYACRYLONITRILE- GRAFT-CHITOSAN AND THEIR APPLICATION FOR THE REMOVAL OF HEAVY METALS FROM AQUEOUS SOLUTION
}

\author{
J.A. DENA-AGUILAR ${ }^{1}$, J. JAUREGUI-RINCÓN ${ }^{1}$, A. BONILLA-PETRICIOLET

${ }^{1}$ Universidad Autónoma de Aguascalientes, Aguascalientes, México, 20131
${ }^{2}$ Instituto Tecnológico de Aguascalientes, Aguascalientes, México, 20256 \\ ${ }^{2}$ Instituto Tecnológico de Aguascalientes, Aguascalientes, México, 20256
${ }^{3}$ Centro de Investigación en Química Aplicada, Coahuila, México, 25250
}

\begin{abstract}
Aminated copolymers of polyacrylonitrile-graft-chitosan (APANCS) were synthesized via the reaction of copolymers of polyacrylonitrile-graft-chitosan (PAN$g$-CS) with diethylenetriamine in solution. Specifically, copolymers of PAN-g-CS have been obtained via a graft polymerization method using acrylonitrile (AN), chitosan (CS) and a free-radical initiating process with ceric ammonium nitrate (CAN) as initiator under air presence. Superficial structure of these copolymers was modified using amination reactions to introduce amine groups on its polymeric matrix. This polymer (APANCS) was applied in the removal of $\mathrm{Pb}^{2+}, \mathrm{Cd}^{2+}$ and $\mathrm{Zn}^{2+}$ ions in aqueous solutions. Samples of PAN- $g$-CS and APANCS were characterized by FT-IR spectroscopy, scanning electron microscopy/energy-dispersive X-ray spectroscopy, TGA, DSC and gravimetric analyses. Results of this study suggest that the amine groups of the structure of chitosan are involved in the grafting process onto the polymeric matrix of polyacrylonitrile. On the other hand, the adsorption capacities for heavy metal removal of this polymer were significantly improved using the chemical modification with amination reactions. Specifically, this polymeric material showed a maximum adsorption of $12.93,2.27$ and 2.10 $\mathrm{mg} / \mathrm{g}$ at $\mathrm{pH} 5$ for the removal of $\mathrm{Pb}^{2+}, \mathrm{Cd}^{2+}$ and $\mathrm{Zn}^{2+}$ ions, respectively.
\end{abstract}

Keywords: Polyacrylonitrile, chitosan, heavy metal removal, graft polymerization.

\section{INTRODUCTION}

The presence of heavy metal ions in water bodies implies both environmental and public health problems because these metals are toxic for ecosystems and human beings. Note that heavy metals are considered as accumulative and no biodegradable pollutants (Deng et al., 2003; Li et al., 2005; Chen et al., 2006; Li et al., 2007). During last years, novel materials for environmental and industrial applications have been synthesized from the modification of natural polymers using graft polymerization procedures (Jin and Bai, 2002; Pourjavadi et al., 2003; Li et al., 2005; dos Santos et al., 2006). In particular, the chemical modification of chitosan (CS) with vinyl monomers by graft copolymerization has been studied (Pourjavadi et al., 2003; ZohuriannMehr, 2005; dos Santos et al., 2006; Joshi and Sinha, 2006; Akgün et al., 2007).

CS is a product of the deacetylation of the chitin, which is an abundant natural polymer. The CS has been used in various fields of the human activity because it has interesting properties of biodegradation, biocompatibility and low toxicity (Blackburn, 2004; Kenawy et al., 2005; Rangel-Mendez et al., 2009). This polymer has been also used as an adsorbent for water treatment and it has been studied for the removal of heavy metal ions from aqueous solutions (Rangel-Mendez et al., 2009). In particular, the amine $\left(\mathrm{NH}_{2}\right)$ and hydroxyl $(\mathrm{OH})$ groups of the chemical structure of $\mathrm{CS}$ can interact with some transition metals for forming chelating compounds (Jin and Bai, 2002; Boddu et al., 2003; Joshi and Sinha, 2006; Sadeghi et al., 2008; Sun et al., 2009). Nevertheless, the employment of CS can be limited by its solubility in slightly acid solutions. In particular, the wastewaters polluted by heavy metal ions may show low values of $\mathrm{pH}($ e.g., $\mathrm{pH}<5)$ and, therefore, materials resistant to the $\mathrm{pH}$ attack should be used for the removal of heavy metals in wastewater treatment.

On the other hand, the polyacrylonitrile (PAN) is a common and commercial low-cost product with good physical properties such as hardness and high chemical resistance (Matyjaszewski et al., 1997; Deng et al., 2003) An important characteristic of this polymer is the presence of nitrile functional groups in its chemical structure. PAN is a versatile polymer used to produce several materials including textile fibers, ultra filtration membranes and carbon fibers (Lu et al., 2004; Kruk et al., 2005). Also, PAN has been used to synthetize novel materials for the removal of some heavy metal ions from aqueous solutions (Deng et al., 2003; Hun-Shin et al., 2004; Anirudhan and Ramachandran, 2008). Based on the fact that PAN can be synthesized using free-radical polymerization processes (Matyjaszewski et al., 1997; Kruk et al., 2005; Leiston-Belanger et al., 2006) and CS can be modified via graft polymerization ( $\mathrm{Li}$ et al., 2005; Zohuriann-Mehr, 2005; dos Santos et al., 2006; Joshi and Sinha, 2006; Zhang et al., 2007), this study has focused on the synthesis of a copolymer of polyacrylonitrile-graft-chitosan (PAN-g-CS) via graft polymerization methods. In particular, a free-radical initiating process using ceric ammonium nitrate (CAN) as initiator, under air presence, has been used for the synthesis of this copolymer. PAN-g-CS has been chemically modified with amination reactions using diethylenetriamine for incorporating amine groups into the surface of the copolymers (APANCS). These modified copolymers have been studied in the removal of heavy metal ions from aqueous solution. In summary, this manuscript reports the methodology for the synthesis and characterization of PAN-g-CS and the application of APANCS for the removal of $\mathrm{Pb}^{2+}, \mathrm{Cd}^{2+}$ and $\mathrm{Zn}^{2+}$ ions from aqueous solutions.

\section{MATERIALS AND METHODS}

\subsection{Chemicals used in this study}

Acrylonitrile (AN) monomer of high purity, chitosan (CS) of practical grade obtained from the shells of crab with a deacetylation degree $\geq 75 \%$, diethylenetriamine, ceric ammonium nitrate (CAN), dimethylformamide and acetic acid were purchased from Sigma-Aldrich and these chemicals were used in this study. Deionized (DI) water and nitrate salts of $\mathrm{Pb}^{2+}, \mathrm{Cd}^{2+}$ and $\mathrm{Zn}^{2+}$ (J.T. Baker) were employed to prepare all the metal solutions for adsorption studies.

\subsection{Graft copolymerization of PAN-g-CS}

All the reactions were performed using a reactor of $250 \mathrm{~mL}$ equipped with a mechanical stirrer and a temperature control system. First, CS was dissolved in an aqueous solution of acetic acid $(5 \% \mathrm{v} / \mathrm{v})$. A solution of CAN was prepared by dissolving $9.85 \times 10^{-4} \mathrm{~mol}$ of ceric ammonium nitrate in $10 \mathrm{~mL}$ of water. The total volume of the aqueous solution was $120 \mathrm{~mL}$ in all experiments. CS solution was added to the reactor and the mixture was heated at a given temperature under constant agitation for $5 \mathrm{~min}$. AN monomer and CAN solution were added to the reactor and the mixture was continuously stirred $(115 \mathrm{rpm})$ at $65^{\circ} \mathrm{C}$ until completing the reaction time (240 min). Table 1 shows the AN/CS molar ratios used in the experiments of this study. The reaction product was precipitated using ethanol, filtered and dried at $55{ }^{\circ} \mathrm{C}$ for $48 \mathrm{~h}$. For the separation of PAN homopolymer, $1.0 \mathrm{~g}$ of the raw product was mixed with $40 \mathrm{~mL}$ of dimethylformamide (DMF) during $24 \mathrm{~h}$ at $55^{\circ} \mathrm{C}$ (Pourjavadi et al., 2003). Then, the solid product was separated from DMF and dried at $55^{\circ} \mathrm{C}$ for $72 \mathrm{~h}$ until the solvent was totally removed. Later, the product was washed using a $1 \mathrm{M}$ acetic acid solution at $35^{\circ} \mathrm{C}$ for $24 \mathrm{~h}$ to remove the unmodified and soluble low-molecular-weight CS (Singh et al., 2005). The final product was washed with water until obtaining the neutrality and it was finally dried at $55^{\circ} \mathrm{C}$ for $48 \mathrm{~h}$ until reaching a constant weight. This purified solid (PANg-CS) was considered as the evidence of grafting (Sugahara and Ohta, 2001; Pourjavadi and Zohuriaan-Mehr, 2002; Pourjavadi et al., 2003). A physical mixture of PAN and CS was treated in the same way and it was found that this mixture dissolved completely. Finally, PAN-g-CS was milled to obtain a mean 
particle size of $0.025 \mathrm{~cm}$, which was used for all adsorption experiments. All polymer samples used for the characterization, the chemical modification using amination reactions and the metal adsorption experiments were synthesized using the synthesis conditions for the sample B given in Table 1. In this case, the sample B was synthesized with $94.5 \% \mathrm{w} / \mathrm{w}$ of AN and $5.5 \% \mathrm{w} / \mathrm{w}$ of CS (i.e., $2.14 \mathrm{~mol} \mathrm{x} 10^{-1}$ of AN and $2.34 \mathrm{~mol} \mathrm{x} 10^{-1}$ of CS).

Table 1. Synthesis of PAN-g-CS: AN/CS ratio.

\begin{tabular}{|c|c|c|c|c|c|c|}
\hline \multirow{2}{*}{\multicolumn{2}{|c|}{ Parameter }} & \multicolumn{5}{|c|}{ Sample } \\
\hline & & \multirow{2}{*}{$\begin{array}{c}\mathrm{A} \\
96.0\end{array}$} & \multirow{2}{*}{$\frac{\mathrm{B}}{94.5}$} & \multirow{2}{*}{$\frac{\mathrm{C}}{93.0}$} & \multirow{2}{*}{$\frac{\mathrm{D}}{91.5}$} & \multirow{2}{*}{$\begin{array}{c}\mathrm{E} \\
90.0\end{array}$} \\
\hline \multirow{2}{*}{$\mathrm{AN}$} & $\% \mathrm{w} / \mathrm{w}$ & & & & & \\
\hline & $\mathrm{mol} \times 10^{-1}$ & 2.17 & 2.14 & 2.10 & 2.07 & 2.04 \\
\hline \multirow{2}{*}{ CS } & $\% \mathrm{w} / \mathrm{w}$ & 4.0 & 5.5 & 7.0 & 8.5 & 10.0 \\
\hline & $\mathrm{mol} \times 10^{-6}$ & 1.70 & 2.34 & 2.97 & 3.61 & 4.25 \\
\hline
\end{tabular}

\subsection{Preparation of APANCS}

The synthesis procedure of APANCS was similar to the methodology reported by Deng et al. (2003). Specifically, copolymers APANCS were prepared using a simple one-step reaction by adding $1.0 \mathrm{~g}$ of PAN- $g$-CS into $30 \mathrm{~mL}$ of aqueous solution of diethylenetriamine $(33 \% \mathrm{v} / \mathrm{v})$. This mixture was stirred for $36 \mathrm{~h}$ at $60{ }^{\circ} \mathrm{C}$. Then, copolymers APANCS were separated from the solution and washed with water until obtaining the neutrality. Finally, samples of APANCS were dried at $55^{\circ} \mathrm{C}$ for $48 \mathrm{~h}$ to reach a constant weight. These samples were stored in a desiccator prior to its use in the adsorption studies.

\subsection{Physicochemical characterization of the polymeric materials}

FT-IR spectra were obtained using a Nicolet Magna spectrophotometer 550 with an ATR accessory. Scanning electron micrographs of the superficial morphology of the copolymers were recorded using a scanning electronic microscope (SEM) JSM-7401F JEOL. Samples were covered with goldpalladium before its observation in SEM. An analysis of the sample composition was performed using energy-dispersive X-ray spectroscopy (EDX). The thermal characterization of the samples was performed via a thermogravimetric analysis (TGA) and differential scanning calorimetry (DSC) using a thermogravimetric analyzer TGA Q500 (TA Instruments) and a calorimeter DSC 2920 (TA Instruments), respectively. TGA and DSC analyses were performed from 30 to $600{ }^{\circ} \mathrm{C}$ and from -50 to $250{ }^{\circ} \mathrm{C}$, respectively, at a rate of $10^{\circ} \mathrm{C} / \mathrm{min}$ under a nitrogen flow. Grafting-percentage (\%G) and efficiency $(\% \mathrm{E})$ were calculated based on the weight of products using the following relationships (Jin and Bai, 2002; Singh et al., 2005; Joshi and Sinha, 2006)

$$
\begin{aligned}
& \% \mathrm{G}=\frac{W_{1}-W_{2}}{W_{2}} \times 100 \\
& \% \mathrm{E}=\frac{W_{1}-W_{2}}{W_{3}-W_{2}} \times 100
\end{aligned}
$$

where $W_{1}, W_{2}$, and $W_{3}$ are the weight of pure graft copolymer, CS and raw graft copolymer, respectively.

\subsection{Metal adsorption experiments}

Batch adsorption experiments were performed using PAN- $g$-CS and APANCS. Metal solutions with concentrations from 10 to $100 \mathrm{mg} / \mathrm{L}$ of $\mathrm{Pb}^{2+}, \mathrm{Cd}^{2+}$ and $\mathrm{Zn}^{2+}$ ions were prepared from $\mathrm{Pb}\left(\mathrm{NO}_{3}\right)_{2}, \mathrm{Cd}\left(\mathrm{NO}_{3}\right)_{2} \cdot 4 \mathrm{H}_{2} \mathrm{O}$, $\mathrm{Zn}\left(\mathrm{NO}_{3}\right)_{2} \cdot 6 \mathrm{H}_{2} \mathrm{O}$ and deionized water. Adsorption experiments were performed at $30{ }^{\circ} \mathrm{C}$ and $\mathrm{pH} 5 \mathrm{using}$ an adsorbent/solution ratio of $2.5 \mathrm{~g} / \mathrm{L}$. Preliminary studies showed that the adsorption equilibrium was reached after $120 \mathrm{~h}$. Adsorbent was separated from the solution by filtration and the concentrations of metal ions in the remaining solutions were quantified using a Perkin-Elmer AAnalyst 100 atomic adsorption spectrophotometer. Adsorption capacities $(q$, $\mathrm{mg} / \mathrm{g}$ ) at time $t$ were calculated by a mass balance

$$
q_{t}=\frac{\left(C_{o}-C_{t}\right) V}{m_{s}}
$$

where $C_{o}$ and $C_{t}$ are the initial and final metal concentrations in $\mathrm{mg} / \mathrm{L}$, respectively, $V$ is the volume of the metal solution in $\mathrm{mL}$ and $m_{s}$ is the weight of copolymer used in adsorption experiments given in $\mathrm{g}$.

\section{RESULTS AND DISCUSSION}

\subsection{Analysis of grafting polymerization process and proposed structure} of APANCS

Figure 1 shows the effect of CS concentration on grafting polymerization process. The maximum grafting ratio (i.e., $1574 \% \mathrm{G}$ and $86 \% \mathrm{E}$ ) was observed at a CS concentration of $1.70 \times 10^{-6} \mathrm{~mol}$. Note that both grafting-percentage $(\% \mathrm{G})$ and efficiency (\%E) decreased from 1574 to $520 \%$ and from 86 to $80 \%$, respectively, with the increment of CS concentration. This result indicates that the homopolymer synthesis is favored. This behavior could be attributed to the deactivation of radicals on the growing polymeric chains and by the restricted movement of the radicals due to an increment in the viscosity of reaction medium (Pourjavadi et al., 2003).

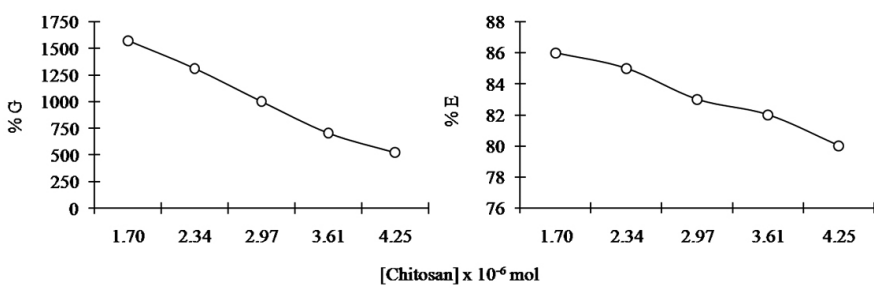

Figure 1. Effect of the amount of the chitosan concentration on the grafting parameters $\% \mathrm{G}$ and $\% \mathrm{E}$. Reactions conditions: i) $\mathrm{CAN}$ concentration: $9.85 \times 10^{-4} \mathrm{~mol}$, ii) concentration of aqueous solution of acetic acid: $5 \% \mathrm{v} / \mathrm{v}$, iii) temperature: $65^{\circ} \mathrm{C}$ and iv) reaction time: $240 \mathrm{~min}$.

On the other hand, Figure 2 shows the chemical structure proposed for the aminated copolymers including the possible interactions between CS, PAN and amination reactions. Results of our study and those obtained in other studies (Kenawy et al., 2005; Pourjavadi et al., 2003; Li et al., 2005; dos Santos et al., 2006) suggest that the CS graft onto the polymeric matrix PAN is performed via the $\mathrm{C} 2$ carbon of the amine group from polysaccharide. Also, the amine groups were incorporated onto the copolymers using the amination reactions by means of the nitrile groups (Deng et al., 2003). As stated, the surface chemistry modification improved the performance of this polymer for the removal of metallic ions.

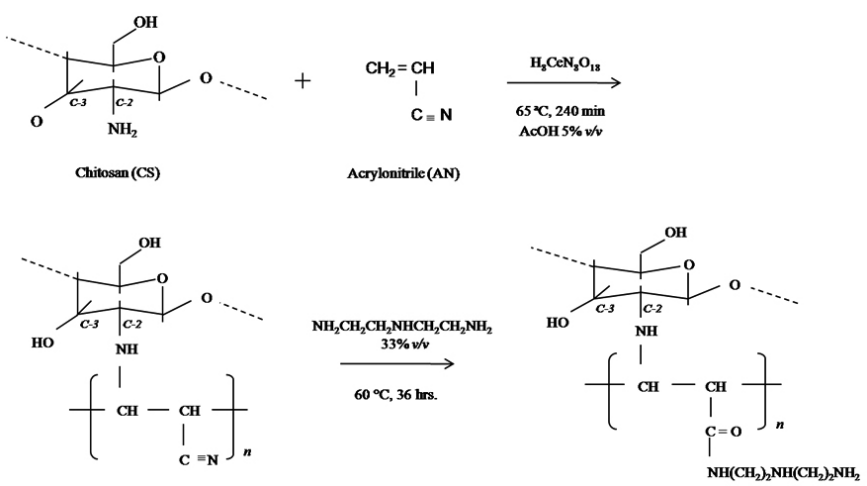

Figure 2. Proposed mechanism for the synthesis of PAN-g-CS and APANCS using the graft copolymerization of CS onto PAN.

\subsection{Results of physicochemical characterization}

FT-IR spectra of CS, PAN, PAN- $g$-CS, and APANCS are given in Figure 3. The principal peaks identified for CS can be assigned as follows: $3380 \mathrm{~cm}^{-1}$ corresponds to $\mathrm{N}-\mathrm{H}$ stretching, $1635 \mathrm{~cm}^{-1}$ is related to bending of $\mathrm{C}=\mathrm{O}$ of amide I, $1580 \mathrm{~cm}^{-1}$ is related to bending of $\mathrm{NH}_{2}$ and peaks at 1080 and 1035 $\mathrm{cm}^{-1}$ are attributed to the saccharide structure (C-O in $\mathrm{COH}$ stretching) ( $\mathrm{Li}$ et al., 2005; Joshi and Sinha, 2006; Zhang et al., 2007; Sadeghi et al., 2008). The principal peak for PAN was identified at $2245 \mathrm{~cm}^{-1}$, which corresponds to the C $\equiv$ N stretching (Deng et al., 2003; Pourjavadi et al., 2003; Singh et al., 2005). PAN-g-CS spectrum presented significant differences with respect to 
the spectra of pure compounds suggesting that a grafting has been achieved. A broad band between $3350-3100 \mathrm{~cm}^{-1}$ was attributed to $\mathrm{NH}$ and $\mathrm{OH}$ groups of the structure of the polysaccharide. Peaks at 2245 and $1455 \mathrm{~cm}^{-1}$ suggest that $\mathrm{C} \equiv \mathrm{N}$ and $\mathrm{CH}-\mathrm{CH}_{2}$ groups are abundantly present in the copolymer. Also, peaks at 1035 and $1080 \mathrm{~cm}^{-1}$ suggest the presence of $\mathrm{C}-\mathrm{OH}$ groups of $\mathrm{CS}$, although, they are of minor intensity. On the other hand, APANCS spectrum presented differences in the intensity of the band at $3350-3100 \mathrm{~cm}^{-1}$ where a more intense band appeared in this spectrum. Peak at $2245 \mathrm{~cm}^{-1}$ suggests that $\mathrm{C}$ $\equiv \mathrm{N}$ groups are abundantly present in the copolymer. Moreover, peak at 1080 $\mathrm{cm}^{-1}$ indicates the presence of $\mathrm{C}-\mathrm{OH}$ groups of CS. This result is attributable to a major presence of amine groups aggregated by the amination reactions. In PAN-g-CS spectrum, the peak at $1580 \mathrm{~cm}^{-1}$ is attributable to $\mathrm{NH}_{2}$ groups, while the APANCS spectrum showed two peaks overlapped at 1635 and $1555 \mathrm{~cm}^{-1}$ attributable to $\mathrm{N}-\mathrm{H}$ groups. This result also suggests a major presence of amine groups in the copolymers aggregated by the amination reactions. In summary, FT-IR analysis showed that graft copolymer and aminated copolymer have been obtained.

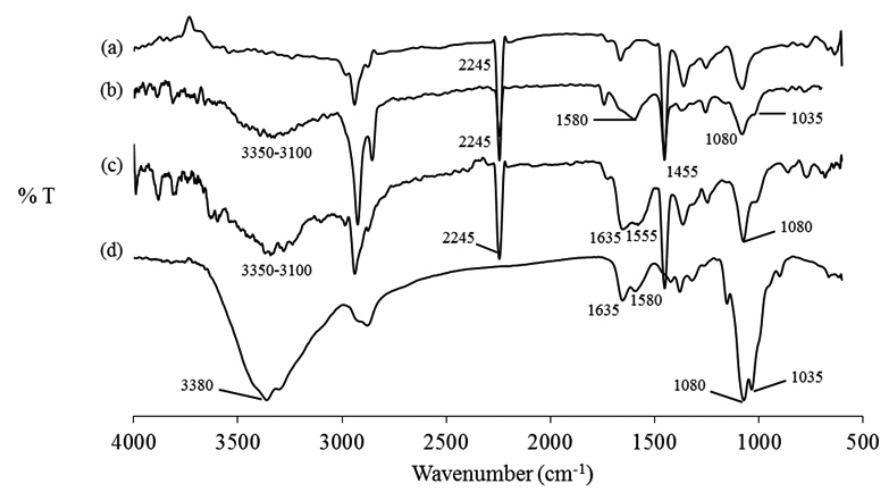
CS.

Figure 3. FT-IR spectra of (a) PAN, (b) PAN-g-CS, (c) APANCS and (d)

Results of EDX analysis of CS, PAN, PAN- $g$-CS, and APANCS are reported in Table 2. Nitrogen percentages of CS (10.82\%), PAN- $g$-CS $(20.39 \%)$ and APANCS $(24.44 \%)$ are attributed to the amine groups present in the polymeric samples. Note that nitrogen percentage in APANCS was higher than those obtained for CS and PAN-g-CS. Therefore, it is expected that this polymer may show a good adsorption performance for heavy metal removal due to these amine groups may interact with these water pollutants. It is convenient to remark that nitrogen percentage of PAN (30.52\%) corresponds to the nitrile groups. Finally, oxygen percentage of CS $(30.89 \%)$ corresponds to groups $\mathrm{OH}$ present in its chemical structure. These results are consistent with the other physicochemical characterization results reported in this study.

Table 2. Results of EDX analysis of polymeric samples.

\begin{tabular}{|c|c|c|c|}
\hline Sample & $\mathrm{C}(\%)$ & $\mathrm{N}(\%)$ & $\mathrm{O}(\%)$ \\
\hline CS & 58.29 & 10.82 & 30.89 \\
\hline PAN & 69.48 & 30.52 & 0.0 \\
\hline PAN-g-CS & 78.14 & 20.39 & 1.47 \\
\hline APANCS & 74.15 & 24.44 & 1.41 \\
\hline
\end{tabular}

Thermal stability and decomposition of CS, PAN and PAN-g-CS were studied using TGA and Figure 4 shows the results of this thermal analysis. The initial degradation temperature of PAN- $g$-CS was higher than those obtained for CS and PAN samples. Primary weight loss started around $300{ }^{\circ} \mathrm{C}$ followed by a total weight loss at $>600^{\circ} \mathrm{C}$. On the other hand, the primary weight loss of CS started around $296{ }^{\circ} \mathrm{C}$ and from 281 to $406{ }^{\circ} \mathrm{C}$ for PAN sample. Weight losses of CS and PAN samples were very drastic (60 and $65 \%$, respectively) at relatively low temperature. PAN- $g$-CS sample showed a $40 \%$ weight loss from 266 to $413{ }^{\circ} \mathrm{C}$. In fact, PAN-g-CS showed a major resistance to the effect of the thermal treatment. Figure 5 shows the DSC scans of CS, PAN and PANg-CS samples under an inert environment. All polymeric samples presented endothermic peaks. PAN and PAN- $g$-CS showed an endothermic peak around $0-50{ }^{\circ} \mathrm{C}$, which can be attributed to water loss. The endothermic maxima in the
DSC curves of CS, PAN and PAN-g-CS were observed at 119.7, 136.8 and $163.8^{\circ} \mathrm{C}$, respectively; the ranges of these peaks are $50-150{ }^{\circ} \mathrm{C}$ for $\mathrm{CS}$ and 80 $200^{\circ} \mathrm{C}$ for PAN and PAN- $g$-CS. The thermal transition can be associated to the endothermic maxima by means of the glass transition temperature $(T g)$ of the polymers. $T g$ of PAN-g-CS was higher than those $T g$ of CS and PAN indicating that the copolymer is more resistant to the effect of the temperature, which is consistent to the TGA results. These results suggest that the reaction of CS with PAN provides a cross-linked structure, which is different from the structure of the starting raw polymers.

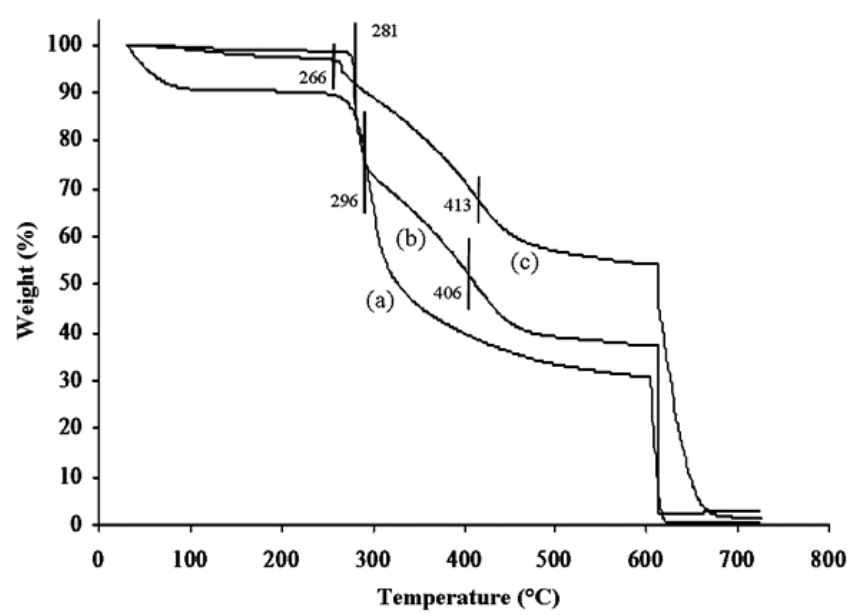

Figure 4. TGA thermograms of polymeric samples: (a) CS, (b) PAN and (c) PAN-g-CS

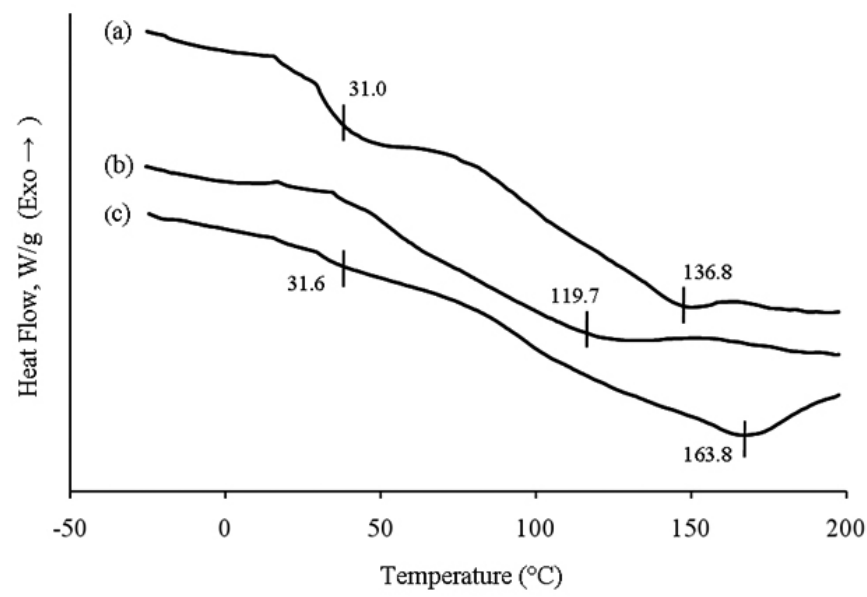

Figure 5. DSC curves of polymeric samples: (a) PAN, (b) CS and (c) PAN$g$-CS.

The morphology of CS, PAN, PAN-g-CS and APANCS samples was studied by SEM, see Figures 6 and 7, respectively. It is clear that the graft copolymerization process affects the surface morphology and also the physicochemical characteristics of the polymer. Figure 6 shows that the flaky nature of CS and the particles agglomerated in shapes of bouquets of PAN were modified due to the reaction of CS with PAN to form cross-linked structures. On the other hand, PAN-g-CS samples showed an irregular structure. Figure 7 shows morphology of the APANCS as a result of the amination reactions where the surface of the APANCS is wrinkled. It is expected that the introduction of the amine groups on the surface of APANCS can change the material properties and affect its adsorption properties for the removal of metallic ions (Deng et al., 2003). 


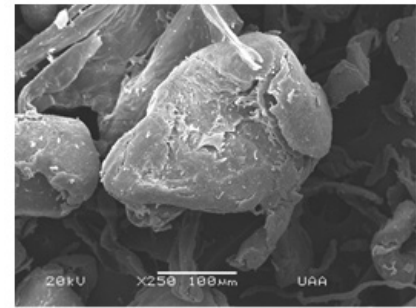

(a)

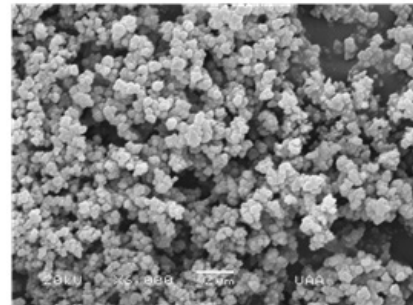

(b)

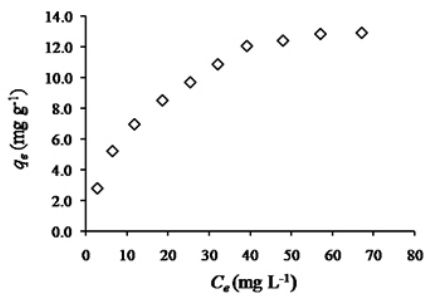

(a)

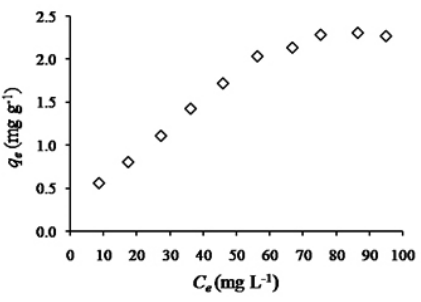

(b)

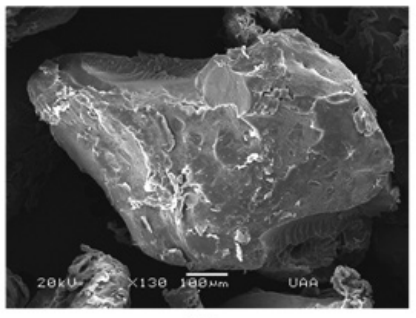

(c)

Figure 6. SEM micrographs of polymeric samples: (a) CS, (b) PAN and (c) PAN-g-CS.

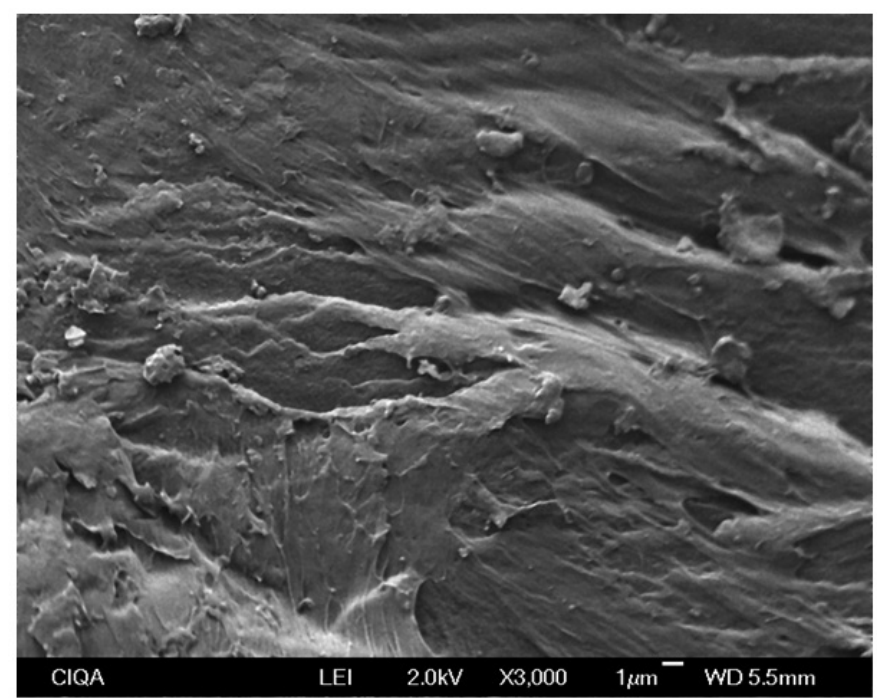

Figure 7. SEM micrographs of APANCS.

\subsection{Adsorption studies of heavy metals}

Adsorption isotherms obtained for the removal of $\mathrm{Pb}^{2+}, \mathrm{Cd}^{2+}$ and $\mathrm{Zn}^{2+}$ ions on APANCS are reported in Figure 8. These isotherms correspond to a Langmuir-type isotherm indicating a favorable adsorption process. With illustrative purposes, Figure 9 shows the maximum adsorption capacities obtained for the removal of $\mathrm{Pb}^{2+}, \mathrm{Cd}^{2+}$ and $\mathrm{Zn}^{2+}$ ions using PAN-g-CS and APANCS. APANCS showed higher adsorption capacities $(q)$ than those obtained for PAN-g-CS for all heavy metal ions. In fact, PAN- $g$-CS showed a poor performance for the removal of these water pollutants (i.e., $<0.40 \mathrm{mg} / \mathrm{g}$ ). In contrast, APANCS showed metal uptakes of $12.93,2.27$ and $2.10 \mathrm{mg} / \mathrm{g}$ of $\mathrm{Pb}^{2+}, \mathrm{Cd}^{2+}$ and $\mathrm{Zn}^{2+}$ ions, respectively. These results indicated that the amination reactions improved the adsorption performance of APANCS in $3400 \%$ for $\mathrm{Pb}^{2+}$, $2200 \%$ for $\mathrm{Cd}^{2+}$ and around $800 \%$ for $\mathrm{Zn}^{2+}$ with respect to the results obtained for PAN-g-CS. These results are consistent to other studies, e.g. Deng et al. (2003), where the amination reactions may improve the adsorption properties of polymers by aggregating amine groups onto its surface. In particular, the adsorption of $\mathrm{Pb}^{2+}$ is higher due to the amino group of the copolymer may share a couple of electrons with the metal forming metal-ligand complex (Balanta et al., 2010).

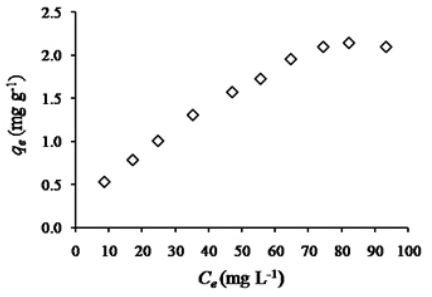

Figure 8. Adsorption isotherms of heavy metal ions on the APANCS: (a) $\mathrm{Pb}^{2+}$, (b) $\mathrm{Cd}^{2+}$ and (c) $\mathrm{Zn}^{2+}$.

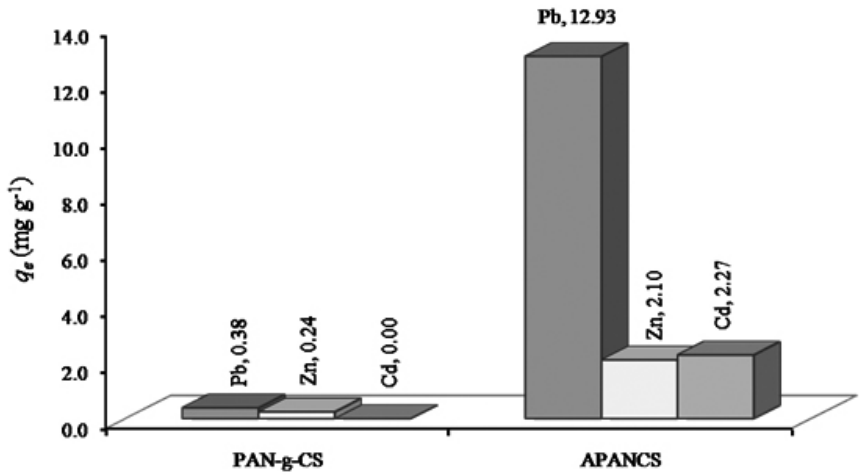

Figure 9. Maximum adsorption capacities for the removal of heavy metal ions on PAN- $g$-CS and APANCS.

\section{CONCLUSIONS}

Synthesis and characterization of PAN-g-CS and APANCS have been reported in this study. Specifically, PAN-g-CS was obtained via graft polymerization methods using $\mathrm{CAN}$ as initiator under air presence. Characterization results indicated that groups $-\mathrm{NH}_{2}$ of $\mathrm{CS}$ and $-\mathrm{CN}$ of PAN were involved in the graft polymerization. On the other hand, modified copolymers (APANCS) have been obtained introducing the amine groups on the surface of the PAN-g-CS by a simple reaction using diethylenetriamine in aqueous solution. Adsorption experiments indicated that APANCS showed higher adsorption capacities for the removal of $\mathrm{Pb}^{2+}, \mathrm{Cd}^{2+}$ and $\mathrm{Zn}^{2+}$ than those obtained for PAN-g-CS. The adsorption mechanics for the removal of these metal ions appears to be related to the formation of metallic complexes with the amine groups of APANCS. It is convenient to remark that PAN-g-CS has potential applications in the textile industry and for the elaboration of biodegradable packing and fibers due to the content of CS and PAN in this copolymer. Finally, APANCS is an attractive polymer for application in water and wastewater treatment due to its physicochemical properties, e.g., thermal resistance and insolubility at acid conditions.

\section{ACKNOWLEDGEMENTS}

This work was supported by the Consejo Nacional de Ciencia y Tecnología of Mexico (CONACYT). We are grateful to the Central Laboratory of Analytical Instrumentation of the Centro de Investigación en Química Aplicada and to the Instituto Tecnológico de Aguascalientes for their valuable support during this study. 


\section{REFERENCES}

1.- Akgün, S.; Ekici, G.; Mutlu, N.; Besirli, N.; Hazer, B.; J. Polym. Res. 2007, 14, 215.

2.- Anirudhan, T. S.; Ramachandran, M.; Ind. Eng. Chem. Res. 2008, 47, 6175.

3.- Balanta, D.; Grande, C. D.; Zuluaga, F.; Rev. Iberoam. Polim. 2010, 11, 297.

4.- Blackburn, R. S.; Environ. Sci. Technol. 2004, 38, 4905.

5.- Boddu, V. M.; Abburi, K.; Talbott, J. L.; Smith, E. S.; Environ. Sci. Technol. 2003, 37, 4449 .

6.- Chen, C.-Y.; Chiang, C.-L.; Chen, C.-R.; Sep. Purif. Technol. 2006, 54 396.

7.- Deng, S.; Bai, R.; Chen, J. P.; Langmuir. 2003, 19, 5058.

8.- dos Santos, K. S. C. R.; Coelho J. F. J.; Ferreira, P.; Pinto, I., Lorenzetti, S. G.; Ferreira, E. I.; Higa, O. Z.; Gil, M. H.; Int. J. Pharm. 2006, 310, 37.

9.- Hun-Shin, D.; Gun-Ko, Y.; Nyon-Kim, W.; Su-Choi, U.; Ind. Eng. Chem. Res. 2004, 43, 2060.

10.- Jin, L.; Bai, R.; Langmuir. 2002, 18, 9765.

11.- Joshi, J. M.; Sinha, V. K.; J. Polym. Res. 2006, 13, 387.

12.- Kenawy, E.-R.; Abdel-Hay, F. I.; El-Magd, A. A.; Mahmoud, Y.; J. of Bioact. and Compat. Polym. 2005, 20, 95.

13.- Kruk, M.; Dufour, B.; Celer, E. B.; Kowalewski, T.; Jaroniec, M.; Matyjaszewski, K.; J. Phys. Chem. 2005, 19, 9216.
14.- Leiston-Belanger, J. M.; Penelle, J.; Rusell, T. P.; Macromolecules. 2006, 39, 1766.

15.- Li, N.; Bai, R.; Liu, C.; Langmuir. 2005, 21, 11780.

16.- Li, F.; Du, P.; Chen, W.; Zhang, S.; Anal. Chim. Acta. 2007, 585, 211.

17.- Lu, A.; Kiefer, A.; Schmidt, W.; Schüth, F.; Chem. Mater. 2004, 16, 100.

18.- Matyjaszewski, K.; Jou, S. M.; Paik, H.-J.; Gaynor, S. G.; Macromolecules. 1997, 30, 6398.

19.- Pourjavadi, A.; Zohuriaan-Mehr, M. J.; Starch/Stärke. 2002, 54, 140.

20.- Pourjavadi, A.; Mahdavinia, G. R.; Zohuriann-Mehr, M. J.; Omidian, H.; J. Appl. Polym. Sci. 2003, 88, 2048.

21.- Rangel-Mendez, J. R.; Monroy-Zepeda, R.; Leyva-Ramos, E.; DiazFlores, P. E.; Shirai, K.; J. Hazard. Mater. 2009, 162, 503.

22.- Sadeghi, A. M. M.; Amini, M.; Avadi, M. R.; Siedi, F.; Rafiee-Tehrani, M.; Junginger H. E.; J. of Bioact. and Compat. Polym. 2008, 23, 262.

23.- Singh, V.; Tripathi, D. N., Tiwari, A.; Sanghi, R.; J. Appl. Polym. Sci. 2005, $95,820$.

24.- Sugahara, Y.; Ohta, T.; J. Appl. Polym. Sci. 2001, 82, 1437.

25.- Sun, B.; Mi, Z.-T.; An, G.; Liu, G.; Zou, J.-J.; Ind. Eng. Chem. Res. 2009, $48,9823$.

26.- Zhang, J.; Wang, L.; Wang, A.; Ind. Eng. Chem. Res. 2007, 46, 2497.

27.- Zohuriann-Mehr, M. J. Iran. Polym. J. 2005, 14, 235. 